\title{
Bariatric Surgery and Diabetes
}

\author{
Konstantinos Spaniolas, John Pender, Emily R. Cunningham, and Walter J. Pories
}

\section{Introduction}

T T WAS TOO GOOD TO BELIEVE. How could a simple change in the plumbing of the gut cure diabetes, the most costly and deadly disease of the industrialized world? And, at the same time, reverse other expressions of the metabolic syndrome, including severe obesity, hypertension, dyslipidemias, asthma, nonalcoholic steatotic hepatitis (NASH), gastroesophageal reflux disease (GERD), and even polycystic ovary syndrome? (Table 1) (R. Blackstone, personal communication, 2009). There were even claims that bariatric surgery operations reduced the prevalence of solid cancers by $70 \%$ within 5 years (1-3).

Yes, it was too good to believe. And it was appropriate for the medical community to demand prospective, randomized, controlled studies published in the most respected, highimpact journals. This year that demand has been met and, yes, the claims are true. This article will review those reports and also ask why, in spite of the overwhelming evidence, less than $1 \%$ of those patients who could benefit from the surgery have access to this life-saving therapy.

Table 1. Remission of Metabolic Syndrome Comorbidities After Bariatric Surgery (In Percent) (Blackstone)

\begin{tabular}{lccc}
\hline Co-Morbid Disease & $\begin{array}{c}\text { Gastric } \\
\text { Bypass }\end{array}$ & Revision & $\begin{array}{c}\text { Adjustable } \\
\text { Gastric Band }\end{array}$ \\
\hline Type 2 Diabetes & 80.5 & 66.7 & 78.6 \\
Hypertension & 63.3 & 50.0 & 35.1 \\
Obstructive Sleep Apnea & 68.9 & 80.0 & 59.2 \\
GERD & 87.6 & 73.9 & 54.5 \\
Venous Insufficiency & 71.0 & 78.9 & 60.4 \\
Asthma & 66.0 & 83.3 & 37.5 \\
Stress Incontinence & 84.0 & 75.0 & 57.1 \\
Depression & 31.4 & 33.3 & 27.2 \\
Osteoarthritis & 67.1 & 50.0 & 49.3 \\
Hyperlipidemia & 61.4 & 50.0 & 25.0 \\
\hline
\end{tabular}

\section{CLINICAL STUDIES}

Bariatric surgery versus intensive medical therapy for diabetes-3-year outcomes

Schauer $P R^{1}$, Bhatt $D L^{2}$, Kirwan $J P^{3}$, Wolski $K^{4}$, Brethauer $S A^{1}$, Navaneethan $S D^{5}$, Aminian $A^{1}$,
Pothier $\mathrm{CE}^{4}, \mathrm{Kim}_{\mathrm{ESH}}^{4}$, Nissen $\mathrm{SE}^{4}$, Kashyap $\mathrm{SR}^{6}$; STAMPEDE Investigators

${ }^{1}$ Bariatric and Metabolic Institute; ${ }^{2}$ Brigham and Women's Hospital, Heart and Vascular Center and Harvard Medical School, Boston, MA; ${ }^{3}$ Lerner Research Institute; ${ }^{4}$ Heart and Vascular Institute; ${ }^{5}$ Urological and Kidney Institute, Cleveland Clinic, Cleveland, $\mathrm{OH}$; and ${ }^{6}$ Endocrinology Institute, Cleveland, $\mathrm{OH}$

N Engl J Med 2014; 370: 2002-13

\section{Background}

Even though Pories et al. (4) reported in 1998 that the gastric bypass produced durable remission of diabetes in $121 / 146$ patients, 9.4 years after surgery, with a $95 \%$ followup, the finding was doubted since it was a clinical series and not a controlled prospective randomized trial. This study by Schauer, published in the NEJM, a high-impact journal, closed this important gap.

\section{Methods}

Outcomes were assessed 3 years after the randomization of 150 obese patients with uncontrolled type 2 diabetes, in a single-center study, to receive either (a) intensive medical therapy alone or (b) intensive medical therapy plus Rouxen-Y gastric bypass or (c) intensive medical therapy and sleeve gastrectomy. The primary end point was a glycated hemoglobin $(\mathrm{HbA} 1 \mathrm{c})$ level of $6.0 \%$ or less. The mean $( \pm \mathrm{SD})$ age of the patients at baseline was $48 \pm 8$ years, $68 \%$ were women, the mean baseline $\mathrm{HbAlc}$ level was $9.3 \% \pm 1.5 \%$, and the mean baseline body mass index (the weight in kilograms divided by the square of the height in meters) was $36.0 \pm 3.5$. A total of $91 \%$ of the patients completed 36 months of follow-up.

\section{Results}

At 3 years, $5 \%$ of the medical therapy group, $38 \%$ of the gastric bypass group $(p<0.001)$, and $24 \%$ of the gastric sleeve group ( $p=0.01)$ reached HbA1c levels of $6.0 \%$ or less. The use of glucose-lowering medications, including insulin, was

Department of Surgery, Brody School of Medicine, East Carolina University, Greenville, NC. 
lower in the surgical groups than in the medical-therapy group. Patients in the surgical groups had greater mean percentage reductions in weight from baseline, with reductions of $24.5 \% \pm 9.1 \%$ in the gastric-bypass group and $21.1 \% \pm 8.9 \%$ in the sleeve-gastrectomy group, as compared with a reduction of $4.2 \% \pm 8.3 \%$ in the medical-therapy group ( $p<0.001$ for both comparisons). Quality-of-life measures were significantly better in the two surgical groups than in the medical-therapy group. There were no major late surgical complications.

\section{Conclusion}

Among obese patients with uncontrolled type 2 diabetes, 3 years of intensive medical therapy plus bariatric surgery was significantly more effective than medical therapy alone as reflected by body weight, $\mathrm{HbA} 1 \mathrm{c}$, use of glucose-lowering medications, and quality of life.

\section{Comment}

The importance of this study is sharply magnified by the observation period of 3 years.

\section{Bariatric surgery versus conventional medical therapy for type 2 diabetes}

Mingrone $G^{1}$, Panunzi $S^{2}$, De Gaetano $A^{2}$, Guidone $C^{l}$, Iaconelli $A^{1}$, Leccesi $L^{1}$, Nanni $G^{3}$, Pomp $A^{4}$, Castagneto $M^{3}$, Ghirlanda $G^{1}$, Rubino $F^{3,4}$

${ }^{1}$ Departments of Internal Medicine and ${ }^{3}$ Surgery, Università Cattolica S. Cuore, Rome, Italy; ${ }^{2}$ National Research Council of Italy-Institute of Systems Analysis and Computer Science (IASI), BioMatLab, Rome, Italy; and ${ }^{4}$ Section of Gastrointestinal Metabolic Surgery, Weill Medical College of Cornell University, New York Presbyterian Hospital, New York, NY

N Engl J Med 2012; 366: 1577-85

\section{Background}

In contrast to Schauer's article, noted above, this study compared conventional medical therapy with (a) the gastric bypass operation (RYGB) and (b) pancreaticobiliary diversion with duodenal switch (DS).

\section{Methods}

In this single-center, nonblinded, randomized, controlled trial, 60 patients between the ages of 30 and 60 years with a $\mathrm{BMI} \geq 35$, a history of at least 5 years of diabetes, and an HbA1c of $7.0 \%$ or more were randomly assigned to receive conventional medical therapy or undergo either RYGB or DS. The primary end point was the rate of diabetes remission at 2 years $(\mathrm{FBS}<100 \mathrm{mg} / \mathrm{dc}$, and $\mathrm{Hb} 1 \mathrm{Ac}$ of $<6.5 \%$ ) in the absence of pharmacologic therapy.

\section{Results}

At 2 years, diabetes remission had occurred in no patients in the medical-therapy group versus $75 \%$ in the RYGB group and $95 \%$ in the DS group ( $p<0.001$ for both comparisons). The average HbA1c level of $8.65 \% \pm 1.45 \%$ decreased in all groups to $7.69 \% \pm 0.57 \%$ in the medical-therapy group, $6.35 \% \pm 1.42 \%$ after RYGB and $4.95 \% \pm 0.49 \%$ after DS.

\section{Conclusion}

In severely obese patients, bariatric surgery resulted in better glucose control than medical therapy. Preoperative BMI and weight loss did not predict the level of improvement.

\section{Comment}

This article, published in the same issue of the NEJM as Schauer's original article, not only corroborated the Cleveland Clinic group's finding but also showed that the DS has even better outcomes than the RYGB in terms of weight control and remission of diabetes. However, the procedure carries a greater 90-day operative mortality $(1 \%)$ versus the gastric bypass $(0.5 \%)$ and higher rates of late complications, including malnutrition and neuropathies. In the United States and the rest of the world, the most commonly performed bariatric operation continues to be RYGB, followed by the GS, and then the adjustable gastric band (AGB) and DS.

\section{Roux-en-Y gastric bypass vs intensive medical management for the control of type 2 diabetes, hypertension, and hyperlipidemia: the Diabetes Surgery Study randomized clinical trial}

Ikramuddin $S^{1}$, Korner $J^{2}$, Lee $W^{3}$, Connett $J^{4}$, Inabnet $\mathrm{WB}^{5}$, Billington $\mathrm{CJ}^{6}$, Thomas $A J^{4}$, Leslie $D B^{1}$, Chong $K^{7}$, Jeffery $R W^{8}$, Ahmed $L^{9}$, Vella $A^{10}$, Chuang $L M^{11}$, Bessler $M^{9}$, Sarr $M G^{12}$, Swain $J M^{13}$, Laqua $P^{14}$, Jensen $M D^{10}$, Bantle $J P^{6}$

${ }^{I}$ Department of Surgery, University of Minnesota, Minneapolis, MN; ${ }^{2}$ Division of Endocrinology, Department of Medicine, Columbia University Medical Center, New York, NY; ${ }^{3}$ Surgery Department, National Taiwan University Hospital, Taipei City, Taiwan; ${ }^{4}$ Division of Biostatistics, School of Public Health, University of Minnesota, Minneapolis, MN; ${ }^{5}$ Division of Metabolic, Endocrine and Minimally Invasive Surgery, Mount Sinai Medical Center, New York, NY; ${ }^{6}$ Division of Endocrinology \& Diabetes, Department of Medicine, University of Minnesota, Minneapolis, MN; ${ }^{7}$ Department of Endocrinology, Min-Sheng General Hospital, Taoyuan City, Taiwan; ${ }^{8}$ Division of Epidemiology \& Community Health, School of Public Health, University of Minnesota, Minneapolis, MN; ${ }^{9}$ Department of Surgery, Mount Sinai Medical Center, New York, NY; ${ }^{10}$ Division of Endocrinology \& Diabetes, Department of Medicine, Mayo Clinic, Rochester, MN; ${ }^{11}$ Division of Metabolism and Endocrinology, Internal Medicine Department, National Taiwan University Hospital, Taipei City, Taiwan; ${ }^{12}$ Department of Gastroenterologic and General Surgery, Mayo Clinic, Rochester, MN; ${ }^{13}$ Scottsdale Healthcare Bariatric Center, Scottsdale, AZ; and ${ }^{14}$ Berman Center for Clinical Research, University of Minnesota, Minneapolis, MN

JAMA 2013; 309: 2240-49

\section{Background}

This prospective, randomized, and multidisciplinary study was conducted in four academic teaching hospitals in the 
United States and Taiwan with five participating surgeons. This study involved 120 participants with a hemoglobin HbA1c level of $8.0 \%$ or higher, BMI between 30.0 and 39.9, $C$ peptide level of more than $1.0 \mathrm{ng} / \mathrm{mL}$, and type 2 diabetes for at least 6 months.

\section{Methods}

All 120 patients received the intensive lifestyle-medical management protocol and 60 were randomly assigned to undergo RYGB. Medications for hyperglycemia, hypertension, and dyslipidemia were prescribed according to protocol and surgical techniques that were standardized.

\section{Results}

After 12 months, 28 participants (49\%) in the RYGB and $11(19 \%)$ in the lifestyle-medical management group achieved the composite goal, that is, HbA1c less than $7.0 \%$, low-density lipoprotein cholesterol less than $100 \mathrm{mg} / \mathrm{dL}$, and systolic blood pressure less than $130 \mathrm{~mm} \mathrm{Hg}$. Participants in the RYGB group required 3.0 fewer medications and lost $26.1 \%$ of their initial body weight compared with $7.9 \%$ from the lifestyle-medical management group. Regression analyses indicated that achieving the composite end point was primarily attributable to weight loss. There were 22 serious adverse events in the gastric bypass group, including 1 cardiovascular event, and 15 in the lifestyle-medical management group. There were four perioperative complications and six late postoperative complications. The gastric bypass group experienced more nutritional deficiency than the lifestyle-medical management group. There were no deaths in either group.

\section{Conclusion}

In mild to moderately obese patients with type 2 diabetes, the addition of RYGB to lifestyle changes and intensive medical management increased the chances of attaining better control of diabetes, dyslipidemia, and hypertension. RYGB patients had fewer medications but more complications.

\section{Comment}

This article supports the conclusions of the previous two reports and, similar to the Schauer article, involves patients with lower $\mathrm{BMI}<35$ and documents that the RYGB is also effective in patients who are not severely obese. This is an important observation because patients with a $\mathrm{BMI} \leq 35$ are now denied access to the RYGB, GS, and DS operations due to the guidelines adopted by Medicare and most private carriers.

These results are not surprising. Obesity and diabetes are not the same disease. The BMI is not a reliable indicator of adiposity, and only one-third of our severely obese patients are diabetic; 1 out of 10 diabetic patients is lean. Furthermore, the outcomes of bariatric surgery in a series of 29 Asian patients with a BMI $<27 \mathrm{~kg} / \mathrm{m}^{2}$ are most convincing. In that series, Malapan et al. (5) concluded that "all clinical and biochemical parameters, except uric acid, were significantly improved." Diabetes mellitus remission was achieved in 11 patients $(37.9 \%)$. There are some data suggesting that the remission rates are lower in leaner patients.

The BMI should no longer be used to determine whether a patient can have bariatric surgery. The BMI, a simple relationship of weight to height $\left(\mathrm{kg} / \mathrm{m}^{2}\right)$, may be a great epidemiologic tool for estimating obesity in a large population, but it fails critically in the assessment of individual patients. The formula does not account for fitness, age, gender, and race. For example, an individual at our university who was $5^{\prime} 8^{\prime \prime}(1.72 \mathrm{~m})$ and weighed 308 lbs $(140 \mathrm{~kg}$ ) with a BMI of 47 (who would qualify for bariatric surgery easily with any U.S. carrier) was our fastest running back on the football team. The fact that the BMI is unigender, ignoring the marked differences in body composition between men and women, speaks for itself. The BMI also ignores the increased deposits of fat in muscle, that is, "marbling." The worst problem with using the BMI, however, is that it is discriminatory, preventing Asian and African American women from access to bariatric surgery. Members of these racial groups develop the comorbidities of severe obesity with lower BMIs than their Caucasian counterparts (6).

\section{Physicians' attitudes about referring their type 2 diabetes patients for bariatric surgery}

Sarwer $D B^{1,2}$, Ritter $S^{l}$, Wadden $T A^{l}$, Spitzer $J C^{l}$, Vetter $\mathrm{ML}^{3}$, Moore $\mathrm{RH}^{4}$

Departments of ${ }^{1}$ Psychiatry, ${ }^{2}$ Surgery, ${ }^{3}$ Medicine, and ${ }^{4}$ Biostatistics and Epidemiology, Perelman School of Medicine, University of Pennsylvania, Philadelphia, PA

Surg Obes Relat Dis 2012; 8: 381-86

\section{Background}

The objective was to investigate physician attitudes about referring patients with type 2 diabetes for bariatric surgery.

\section{Methods}

The study groups included 339 physicians, 142 at an academic medical center and 197 community-based who practiced in specialties likely to treat type 2 diabetes.

\section{Results}

In 93 surveys, a response rate of 27, respondents reported positive impressions of bariatric surgery as a treatment for obesity and type 2 diabetes (79.6\% and $67.4 \%$, respectively) for patients with a BMI $>30-34.9 \mathrm{~kg} / \mathrm{m}^{2}$. Only $20.8 \%$ were willing to refer patients with a $\mathrm{BMI} \leq 35$.

\section{Conclusion}

Although the queried physicians who treat patients with type 2 diabetes had favorable impressions about bariatric surgery as a treatment for obesity and type 2 diabetes, only a few were willing to refer their patients with type 2 diabetes and a body mass index of $30-34.9 \mathrm{~kg} / \mathrm{m}^{2}$ "to randomized research trials of bariatric surgery." 


\section{Comment}

This article reflects the quandary of bariatric surgery: why are patients not being referred in far larger numbers, given the severity and costs of severe obesity, diabetes, and the associated diseases?

\section{Summary}

As this year's review indicates, we now have ample level 1 evidence, from prospective randomized clinical trials in our most respected journals, that surgery produces significantly better outcomes than even the most intensive medical therapies-that is, the ideal that is clearly not feasible in daily medical practice. The operations are as safe as routine cholecystectomies with 90-day mortality rates of $0.3 \%$ with lengths of stay of 1-2 days. As in other operations, there are variations in outcomes such as weight loss and rates of remission with gender, age, duration of disease, use of insulin, and other factors. In the NIH-funded study, the Longitudinal Assessment of Bariatric Surgery (LABS) (7), none of the patients returned to their original weight in 3 years and only $2.1 \%$ of the cohort fell in the quartile that lost the least weight.

Bariatric surgery, again, similar to other operations, has its share of late complications, including marginal ulcers, episodic hypoglycemic attacks, neuropathies, and emotional issues. However, even these compare well to strokes associated with type 2 diabetes.

Given this evidence, how can we explain the leveling of bariatric surgery at about 200,000 per year, about $1 \%$ of the population who would benefit from the operations? If there were a pill that could produce full and durable remission of type 2 diabetes in four out of five patients, with significant improvement in the rest and a reduction in mortality of $83 \%$, would we have the same response or would the demand outstrip our ability to produce it?

History suggests that delays in the adoption of new approaches in medicine are not new. Even though Sir Humphry Davy noted in 1799 that "as nitrous oxide in its extensive operation appears capable of destroying physical pain, it may probably be used with advantage during surgical operations in which no great effusion of blood takes place," anesthesia was not adopted until after the demonstration by William T.G. Morton in 1846, a delay of 47 years. Alexis Carrel described the principles of vascular suture in 1894, transplantation of organs in 1905, placement of a carotid venous patch in 1906, tissue culture in 1912, and a cardiac perfusion pump in 1935, and even though these advances were described in the best journals and he was awarded the Nobel Prize, introduction into medical practice was very slow. The first repair of an aortic aneurysm at the University of Rochester was performed in 1961 (a delay of 67 years), the first kidney transplant by Joseph Murray was reported in 1954 (a delay of 49 years), Charles Rob published the first carotid endarterectomy in 1954 (a delay of 49 years), and John Gibbon carried out the first open-heart operation in a woman in 1943 (a delay of 8 years).
Nor are these delays limited to medicine. The Chinese invented black powder in the ninth century; Nobel patented dynamite in 1867. Einstein introduced his formula $E=M C^{2}$ in 1905; it took the pressure of a world war and 40 years to discover atomic fusion. Turing invented the first real computer in 1936; Gates founded Microsoft in 1976. On the other hand, the adoption of flight progressed much more rapidly. The first successful flight by the Wright brothers was in 1903; the first airline was founded by Thomas Henry David in 1916.

In summary, for the first time, we have a safe, effective, and durable treatment for diabetes and the other expressions of the metabolic syndrome. Only time will tell whether there will be wider application of these operative procedures or whether better medications make the surgery obsolete. In the meantime, bariatric surgery offers remarkable new approaches for research, the study of patients first with diabetes and then without it that, hopefully, will allow us to conquer this costly and cruel disease.

\section{Author Disclosure Statement}

W.J.P. received grant funding from $J \& J$ and the NIH. The other authors have no competing financial interests.

\section{References}

1. Christou NV, Lieberman M, Sampalis F, Sampalis JS. Bariatric surgery reduces cancer risk in morbidly obese patients. Surg Obes Relat Dis 2008; 4: 691-95.

2. Adams TD, Stroup AM, Gress RE, Adams KF, Calle EE, Smith SC, Halverson RC, Simper SC, Hopkins PN, Hunt SC. Cancer incidence and mortality after gastric bypass surgery. Obesity (Silver Spring) 2009; 17: 796-802.

3. Sjöström L. Review of the key results from the Swedish Obese Subjects (SOS) trial-a prospective controlled intervention study of bariatric surgery. J Intern Med 2013; 273: 219-34.

4. Pories WJ, Swanson MS, MacDonald KG, Long SB, Morris PG, Brown BM, Barakat HA, deRamon RA, Israel G, Dolezal JM, et al. Who would have thought it? An operation proves to be the most effective therapy for adult-onset diabetes mellitus. Ann Surg 1995; 222: 339-50.

5. Malapan K, Goel R, Tai CM, Kao YH, Chang PC, Huang CK. Laparoscopic Roux-en-Y gastric bypass for nonobese type II diabetes mellitus in Asian patients. Surg Obes Relat Dis 2014 [Epub ahead of print]; doi: 10.1016/j.soard.2014.01.018.

6. Stevens J, Truesdale KP, Katz EG, Cai J. Impact of body mass index on incident hypertension and diabetes in Chinese Asians, American Whites, and American Blacks: the People's Republic of China Study and the Atherosclerosis Risk in Communities Study. Am J Epidemiol 2008; 167: 1365-74.

7. Courcoulas AP, Christian NJ, Belle SH, Berk PD, Flum DR, Garcia L, Horlick M, Kalarchian MA, King WC, Mitchell JE, Patterson EJ, Pender JR, Pomp A, Pories WJ, Thirlby RC, Yanovski SZ, Wolfe BM; Longitudinal Assessment of Bariatric Surgery (LABS) Consortium. Weight change and health outcomes at 3 years after bariatric surgery among individuals with severe obesity. JAMA 2013; 310: 2416-25. 